\title{
A NEW APPLICATION OF THE PRINCIPLE OF COMPOSITE PHOTOGRAPHY TO THE IDENTIFICATION OF HANDWRITING.
}

Francis Galton was the first to point out in fugitive memoirs, and notably in his epoch-making work, "The Human Faculty," that one could sift the common from the accidental features of a number of objects by exposing them to a sensitized plate in such a manner that the similar parts of the different objects should occupy as nearly as possible the same part of the plate, and that each object should be exposed for only a fraction of the length of time necessary to complete a picture on the film used. This fraction depended generally, if not always, on the number of objects and on the sensitiveness of the film. For example, if there were eighteen objects and the plate took thirty-six seconds to develop, each object would ordinarily be exposed for two seconds. It is easy to see that the result in the finished picture would be that those features which all the objects had in common would be re-enforced by each separate exposure, whereas those features which were accidental or variable, and which would be different for every individual, would be exposed for but two seconds and would be so indistinct as practically to fade away. Where the object was to catch a family likeness by exposing all the members male and female to the same portion of the plate, the result is a curious medley of faint whiskers, and moustache; of hair parted in the middle and at the side; of female gowns with buttons to the throat and of male shooting jackets thrown open. But out of all this faint halo of confusion and blur, there starts a characteristic face which is the family type. Very often, too, this type face resembles noticeably two different members of a family between whom no one can find a resemblance. It is this latter fact (which might have been expefted) that induced me to look to the process for aid in resolvin the difficult problem of identity of origin in handwriting. When a number of animals of the same race are thus treated, the method secures the fixing of the race or family characteristics, etc., as the case may be. When a number of pictures or ccins bearing different representations of the same individual or scene are the objects, the result is to obtain either the average appearance of the same thing under different conditions (as for instance a man at different times of 
lik: or the average of the impression made by identically the same thins on different artists. In this case, the merit of the process is that it constructs its image out of all that many pairs of trained eyes have seen, without giving undue weight to any one pair. So far then, these efforts have been directed to re-finding a lost or concealed existence through multiple testimony, very much as the law tries to get at the truth by examining a number of witnesses.

The case of handwriting is, however, a different one. With a given mental image before one of what one desires to write; and with a given relation of will-power, nerve sensitiveness and muscular force, the same signature could be repeated a thousand times, provided that all these conditions were invariable, and no others were superadded. So far from this being the case, however, every one of the factors just named, which produce a signature depends on physical and mental -in other words, on extraneous influences, to a very large degree. A desire to make an up stroke, and the movement commenced to effect this, is met by an unexpected obstacle in the paper, a slight twinge in the shoulder, or a sudden noise, and the projected line would show (were we sufficiently cognizant of the detailed working of all the complicated parts of our mental machinery) just the order in which the different sentient and executive parts of us had been affected, and to what extent. But while these ever-recurring accidents result in preventing the signature from ever being made exactly as intended, * the fact that no two of them effect the same kind or amount of deviation leaves it in the power of the experimenter to extract from this process the "ideal" signature-a signature which probably never was seen as it appears, and yet which so combines all the visible results of a particular will acting on a particular arm to trace a known design with a pen or pencil on paper, that it may justly be called the type signature of that writer. What was said of the resemblance of every object of a group to the composite made of that group (provided the objects chosen have any claim to be so associated), while often differing widely from other members of the same group, is true of handwriting. It has been remarked that the composite signature is an ideal, and never was realized. This is because the lines along which the strongest

* The word "intended" is used to imply the effect of the will through the hand if not modified by these accidents, and not conscious intention. 
reenforcement is made are those where locally varying deviations most frequently cross. To put it in another form, suppose the lines $a, b, c$ and $d$ to be in agreement as follows: At the point $a^{\prime}, b$ does not cross, but $c$ and $d$ do. At $b^{\prime}, c$ does not cross, but $d$ and $a$ do. At $c^{\prime}, d$ does not cross, but $a$ and $b$ do. The line which, without very minute inspection, would represent to the eye part of the ideal signature, would be that traced through the points $a^{\prime}, b^{\prime}, c^{\prime}, d^{\prime}$, because those points having superposed lines of three out of the four signatures would be darker, while the variations at each of these points would be indistinct.

In examining with care a composite signature as just described, it at once arrests the attention that the variations are not equally distributed over the entire body of the letter, but that there are regions of each letter where variation of a particular kind is noticeable, and other regions where there is little or none. The more writings of an individual are compared the more forcibly does this fact appear, until finally one is tempted to conclude that after a handwriting is once formed, it cannot naturally exhibit deviations except within a defined variation and in certain limited areas adjacent to the separate letters. It is thus as great an assistance to the observer to study the variations as to study the ideal signature. Indeed, the variations are all important in the matter of identification, and if there were no variations the method would be inapplicable. A comparatively small number of signatures will give the maximum and minimum of variation in any given region of one of the letters forming it. Moreover, the kind of variation is easily observed where there are a number together, so that the most perfect adept at forgery could hardly hope to simulate the microscopically minute characteristics of variations which are simply the visible expression of a series of indefinitely complex relations of muscle and nerve.

In a case which was recently brought before the Orphans' Court in this city, this principle of composite photography was for the first time applied by me to the purpose of identifying handwriting, and from the experience thus far gained, it is thought that it will better accomplish the object than will the mere opinions of the most experienced experts.

PERSIFOR FrAZER.

Philadelphia, Fanuary I9, 1886 . 